\title{
Charged black holes in effective string theory
}

\author{
S. Mignemi ${ }^{\dagger}$ and N. R. Stewart \\ Laboratoire de Physique Théorique, Gravitation et Cosmologie Relativiste \\ Université de Paris VI, CNRS/URA 769 \\ Tour 22, 4e étage, B.C. 142 - 4, Place Jussieu, 75252 Paris Cedex 05, France
}

\begin{abstract}
We investigate the qualitative new features of charged dilatonic black holes which emerge when both the Yang-Mills and Gauss-Bonnet curvature corrections are included in the effective action. We consider these perturbative effects by an expansion up to second order in the inverse string tension on the four dimensional Schwarzschild background and determine the backreaction. We calculate the thermodynamical functions and show that they can behave like those of the Garfinkle-Horowitz-Strominger (GHS) solution [1] or in a more conventional way, depending on the value of the magnetic charge $q$. Moreover, we find that for magnetic charge above a critical value, the temperature of the black hole has a maximum and goes to zero for a finite value of the mass. This indicates that the conventional Hawking evaporation law is modified by string theory at a classical level.
\end{abstract}

November 1992

$\dagger$ Postal address: INFN, Sezione di Cagliari, via Ada Negri 18, 09127 Cagliari, Italy 


\section{Introduction}

It has been recently established [1-3] that the classical black hole solutions of Einstein gravity in four dimensions are endowed with new features when the theory is modified by the introduction of the low energy string corrections to the action. A key property of these modified actions is the non-minimal coupling of the dilaton and axion fields with gravity and other fields. This fact permits to circumvent a class of well-known "no-hair"theorems, which essentially state the triviality of non-minimally coupled scalar fields [4].

In particular, if one neglects the Gauss-Bonnet corrections, exact charged, spherically symmetric solutions with non trivial scalar hair are obtained in ref. [1,5]. These have also been extended to the case of both dyonic and axion charge in the spherically [3] and axially [6] symmetric case. As is well known, if the mass of the black hole is large compared to the Planck mass, then all vacuum solutions of Einstein gravity are approximate solutions of low energy string gravity. The fact that the dilaton field couples to the Yang-Mills field strength means that the charged black hole solutions in string theory differ fundamentally from the classical Reissner-Nordström form. Furthermore, the thermodynamical properties are quite unconventional: the extremal dilaton black holes appear to have zero entropy but non-zero, finite temperature [7]. This behaviour has been interpreted as due to a repulsive potential barrier created by the scalar field, which prevents thermic contact with the exterior regions. It is then argued that these black holes behave much like elementary particles [8]; important consequences ensue for black hole evaporation $[8,9]$.

Garfinkle et al. [1] obtained the exact classical solution for a four dimensional charged black hole. They kept terms of order one in the inverse string tension $\alpha^{\prime}$ in the Yang-Mills field, but not in the curvature. For large mass holes $\left(M \gg M_{P l}\right)$ one may truncate at this order and neglect the same order curvature contributions outside the event horizon. With an exact solution, comes the advantage of making statements about the global properties of the solution. However, from the string theory point of view the low energy effective action consists of an infinite perturbative expansion in $\alpha^{\prime}$. Of course near the singularity the curvature becomes so large that all terms in the $\sigma$-model expansion would be important. But in order to examine the structure of the event horizon, one may consider only regions of finite curvature exterior to that region. Accordingly, in this work we shall consider the perturbative effects up to second order in $\alpha^{\prime}$ of both the Yang-Mills and curvature squared terms on the conventional Schwarzschild background solution. From this point of view, we are considering the $O\left(\alpha^{\prime}\right)$ string theoretic perturbations of Einstein gravity in vacuo. In so doing, we shall determine the backreaction induced by these terms and the dilaton on the geometry and the modified thermodynamics of the model.

We shall adopt units such that $\hbar=c=G=k=1$. So if the black hole is large enough that $\alpha^{\prime} \ll M^{2}$, then the curvature terms can be considered as perturbations about the background solution. In particular we shall see that the Gauss-Bonnet terms contribute to the field equations for the metric only as second order corrections whereas the electric or magnetic terms are $O\left(\alpha^{\prime}\right)$ corrections to the vacuum Schwarzschild background. Thus one can justify the neglect of the Gauss-Bonnet term as a first approximation far from the singularity as in [1].

Before proceeding with our analysis there are some remarks to be made concerning

the form of the effective low energy string theory which we consider: 
(i) The extra internal dimensions of heterotic string theory may be assumed to decouple from the physical 4-dimensional spacetime by considering the topology (4-dimensional spacetime $) \times($ internal compact space $)$ and by imposing appropriate boundary conditions on the internal coordinates. Alternatively, as in the case of Calabi-Yau compactifications, one can "decouple" the internal space by truncating all fields to their zero-modes on the internal manifold. Thus we shall focus our attention on the four dimensional string theory. Moreover, we shall not consider dilaton potentials which could be produced by the effect of dimensional reduction.

(ii) The antisymmetric two-form $B_{m n}$, which arises in the ground state of the string spectrum, appears in the action as the three-form axion field strength $H_{m n p}$ coupled to the dilaton, where

$$
H_{m n p}=\partial_{[m} B_{n p]}+\frac{\alpha^{\prime}}{4 \sqrt{2}}\left(\Omega_{m n p}^{(Y)}-\Omega_{m n p}^{(L)}\right)
$$

$\Omega^{(L)}$ and $\Omega^{(Y)}$ are the Lorentz and Yang-Mills Chern-Simons three-forms, respectively. Notwithstanding the Chern-Simons terms, the non-minimal dilaton coupling to the axion field strength does not alter the stationary spherically symmetric vacuum solutions of Einstein gravity (by a duality transformation $H_{m n p}$ is equivalent to a pseudoscalar and so the uniqueness theorem holds [10]). The situation is changed however for axisymmetric spacetimes where the Lorentz Chern-Simons term contributes to the field equations and thus acts as a source for non trivial dilaton and axion hair [2,11]. Also in the case of dyons with both electric and magnetic charge, the Yang-Mills Chern-Simons form acts as a source for $H_{m n p}$ through the non-zero $F \wedge F$ term [12]. But for spherically symmetric spacetimes, the Lorentz-Chern-Simons term can be expressed as the exterior derivative of a two-form and thus absorbed into the definition of $B_{m n}$ [13]. In the present case we shall "freeze" in the $B_{m n}$ background and discard its role. Since we aim to study the effect of the Gauss-Bonnet term on the charged solutions of refs.[1,5], we shall not be considering the dyonic case and thus the Yang-Mills Chern-Simons term vanishes in our case. For these reasons we shall drop the axion field strength from the action, consistently with the field equations.

The paper is organized as follows: in section 2 we introduce the action and the field equation and discuss the limits of validity of our approximation. In section 3 we discuss the case of zero charge, which presents some interest by itself in the context of the "nohair" theorems of general relativity. In section 4 the perturbative expansion of the GHS solution is studied in a gauge inspired by the exact solution and compared with the exact result. Section 5 deals with the full first order effective action including both the Maxwell and Gauss-Bonnet terms: the approximated solutions are obtained in the same gauge and

their thermodynamical properties are discussed. Finally, in the appendix we rederive the same results in a more conventional gauge.

\section{The field equations}

The bosonic sector of the effective action for the heterotic string is given to leading order in $\alpha^{\prime}$ as:

$$
S_{\text {eff }}=\frac{1}{16 \pi} \int d^{4} x \sqrt{-g}\left[\mathcal{R}-2(\nabla \Phi)^{2}+\alpha \mathrm{e}^{-2 \Phi}\left(\mathcal{S}-F^{2}\right)\right]
$$


where $\alpha \equiv \alpha^{\prime} / 8$ and $\mathcal{S} \equiv \mathcal{R}_{a b c d}^{2}-4 \mathcal{R}_{a b}^{2}+\mathcal{R}^{2}$ is the Gauss-Bonnet term. For the reasons given above, the terms containing the axion have been omitted.

From variation of the action (1) we obtain the following equations of motion:

$$
\begin{gathered}
\mathcal{R}_{m n}=2 \nabla_{m} \Phi \nabla_{n} \Phi+2 \alpha \mathrm{e}^{-2 \Phi}\left(F_{m p} F_{n p}-\frac{1}{4} g_{m n} F^{2}\right) \\
+4 \alpha \mathrm{e}^{-2 \Phi}\left[4 \mathcal{R}_{p(m} \nabla_{n)} \nabla_{p} \Phi-2 \mathcal{R}_{m n} \nabla_{p} \nabla_{p} \Phi-g_{m n} \mathcal{R}_{p q} \nabla_{p} \nabla_{q} \Phi\right. \\
\left.-\mathcal{R} \nabla_{m} \nabla_{n} \Phi+\frac{1}{2} g_{m n} \mathcal{R} \nabla_{p} \nabla_{p} \Phi-2 \mathcal{R}_{q m n p} \nabla_{p} \nabla_{q} \Phi\right] \\
-8 \alpha \mathrm{e}^{-2 \Phi\left[4 \mathcal{R}_{p(m} \nabla_{n)} \Phi \nabla_{p} \Phi-2 \mathcal{R}_{m n} \nabla_{p} \Phi \nabla_{p} \Phi-g_{m n} \mathcal{R}_{p q} \nabla_{p} \Phi \nabla_{q} \Phi\right.} \\
\left.-\mathcal{R} \nabla_{m} \Phi \nabla_{n} \Phi+\frac{1}{2} g_{m n} \mathcal{R} \nabla_{p} \Phi \nabla_{p} \Phi-2 \mathcal{R}_{q m n p} \nabla_{p} \Phi \nabla_{q} \Phi\right] \\
\nabla^{2} \Phi=\frac{\alpha}{2} \mathrm{e}^{-2 \Phi}\left(\mathcal{S}-F^{2}\right) \\
\nabla_{p}\left(\mathrm{e}^{-2 \Phi} F_{p m}\right)=0
\end{gathered}
$$

In order to find approximate solutions to (2) we shall expand the fields around the background constituted by the Schwarzschild metric with vanishing dilaton, which is of course a solution of the field equations. Our expansion will be in the parameter $\alpha$ or, more correctly, in $\frac{\alpha}{m^{2}}, m$ being the mass of the Schwarzschild solution. Since $\alpha$ is believed to be of order unity in Planck units, the expansion is valid for large $m$, in the regions where $\alpha \mathcal{S} \ll \mathcal{R}$, i. e. for $r^{3} \gg \alpha m$. For macroscopic black holes $(m \gg 1)$ this condition is always satisfied, except in a neighborood of the singularity, well inside the horizon (region of strong curvature). Near the physical singularity, however, the higher order corrections to the effective string lagrangian become important and so the perturbation theory is no longer reliable. For charged holes one must also require that $\alpha F^{2} \ll \mathcal{R}$. This condition holds for $r \gg \frac{\alpha q^{2}}{m}$; again, if the charge is not too large, this condition is satisfied by macroscopic black holes far from the singularity.

Alternatively, one might study the effect of the Gauss-Bonnet term by perturbing the exact charged GHS solution. The perturbation would be in the parameter $\frac{q^{2}}{m}$, and would be valid for $F^{2} \gg \mathcal{S}$, i.e. $r \gg m$. The range of validity of this alternative expansion is therefore smaller. Moreover, the physical interpretation as expansion in the string parameter $\alpha^{\prime}$ would be lost. For these reasons we will not adopt this point of view.

\section{The $\mathrm{q}=0$ case}

In this section we study the pure graviton-dilaton system, by making the ansatz $F=0$, consistently with the equations of motion. Besides its interest in connection with string theory, this special case is interesting because it offers an example of non-trivial scalar hair in a purely scalar-gravity theory. The relevance of the Gauss-Bonnet term for gravity is due to the fact that it is the only quadratic lagrangian which gives rise to second order field equations for the metric [14]. Moreover it does not introduce new propagating degrees of freedom into the ghost-free theory [15]. For constant $\Phi$ however, this term does not 
contribute to the field equations, since it is a total derivative in four dimensions. Only its coupling with the dilaton can therefore give rise to non-trivial solutions in four dimensions.

Similar models have been studied in refs.[16-19]. Ref. [16] and [17], however, consider the higher-dimensional case but do not treat in detail the 4-dimensional limit, where the first order corrections due to the Gauss-Bonnet term vanish. In ref. [17] is shown that, in dimensions higher than four, at first order in $\alpha^{\prime}$, the temperature has a maximum and then goes to zero for a finite value of the mass of the holet. This would mean that a stable configuration could be reached during the evaporation of the black hole. However, as we shall see, this is not the case in four dimensions, because then the correction to the temperature has the opposite sign.

The four dimensional case has been discussed in refs. [18,19] in connection with Kaluza-Klein theories; there the relevance of the model for the no-hair theorems was pointed out. However, an essential difference from our treatment is the fact that Poisson [19] does not perform the conformal rescaling which puts the lagrangian in its canonical form. This canonical form should be used for reasons of stability [20] and because it gives an anambiguous definition of the physical mass [17].

We shall look for the spherical symmetric solutions of the field equations (2) by perturbing around the background given by the Schwarzschild solution with vanishing scalar field $\left(\phi_{0}=0\right)$. For vanishing $F$ the scalar field equation reduces to:

$$
\nabla^{2} \Phi=\frac{\alpha}{2} \mathrm{e}^{-2 \Phi} \mathcal{S}
$$

where the Gauss-Bonnet term $\mathcal{S}=\frac{48 m^{2}}{r^{6}}$ for the Schwarzschild background. Expanding $\Phi$ as

$$
\Phi(r)=\alpha \phi_{1}(r)+\alpha^{2} \phi_{2}(r)+\ldots
$$

one has at first order

$$
\left[r(r-2 m) \phi_{1}^{\prime}\right]^{\prime}=\frac{24 m^{2}}{r^{4}}
$$

where the prime denotes the derivative with respect to $r$.

The only solution of (4) regular at the horizon $r=2 m$, is given by (apart from an arbitrary constant which we fix to zero, by requiring $\phi_{1} \rightarrow 0$ at infinity):

$$
\phi_{1}=-\frac{1}{m}\left(\frac{1}{r}+\frac{m}{r^{2}}+\frac{4 m^{2}}{3 r^{3}}\right)
$$

This solution has a dilatonic charge $\frac{\alpha}{m}$, corresponding to a long range repulsive potential. As is usual models of this type, this charge is not a new independent parameter [1].

One can now calculate the backreaction on the metric which is induced by the scalar field, by substituting (5) into the gravitational equations (2a). It is easy to see that,

$\dagger$ Actually, in ref. [17] the Gauss-Bonnet term is replaced by the square of the Riemann tensor. At first order of an expansion around the Schwarzschild background the results are however the same, since $\overline{\mathcal{R}}_{m n}=\overline{\mathcal{R}}=0$ for the Schwarzschild solution. 
contrary to the higher dimensional case [17] the first contribution to the r.h.s. arises only at order $\alpha^{2}$. In fact, in four dimensions, at order $\alpha^{2}$, the r.h.s. of (2a) reduces to

$$
2 \nabla_{m} \phi_{1} \nabla_{n} \phi_{1}-8 \overline{\mathcal{R}}_{b m n a} \nabla_{a} \nabla_{b} \phi_{1}
$$

where $\overline{\mathcal{R}}_{\text {bmna }}$ is the value of the Riemann tensor in the Schwarzschild background.

The static, spherically symmetric metric can now be written as

$$
d s^{2}=-\mathrm{e}^{2 \nu(r)} d t^{2}+\mathrm{e}^{2 \mu(r)} d r^{2}+r^{2} d \Omega^{2}
$$

and the metric functions can be expanded as

$$
\nu(r)=\nu_{0}(r)+\alpha^{2} \tau(r) \quad \mu(r)=\mu_{0}(r)+\alpha^{2} \sigma(r)
$$

with $\mathrm{e}^{2 \nu_{0}}=\mathrm{e}^{-2 \mu_{0}}=1-\frac{2 m}{r}$, corresponding to the Schwarzschild solution; we have taken into account that there are no corrections to the metric functions at order $\alpha$. Substituting for the metric functions $\mu(r)$ and $\nu(r)$ in eqn.(2a), one finds two independent field equations:

$$
\begin{gathered}
\sigma^{\prime}+\tau^{\prime}=r \phi_{1}^{\prime 2}-\frac{8 m}{r^{2}} \phi_{1}^{\prime \prime} \\
\sigma^{\prime}-\tau^{\prime}+\frac{2 \sigma}{r-2 m}=-\frac{8 m}{r^{2}} \phi_{1}^{\prime \prime}+\frac{16 m(r-3 m)}{r^{3}(r-2 m)} \phi_{1}^{\prime}
\end{gathered}
$$

and, upon combining the two,

$$
[(r-2 m) \sigma]^{\prime}=\frac{1}{2} r(r-2 m) \phi_{1}^{\prime 2}+8 m\left[\frac{r-3 m}{r^{3}} \phi_{1}^{\prime}-\frac{r-2 m}{r^{2}} \phi_{1}^{\prime \prime}\right]
$$

We notice from eqn. (9a) that in presence of a scalar field the metric functions $\mu$ and $-\nu$ are not equal.

Substituting (5) in (10) and integrating, one obtains for $\sigma$ :

$$
\sigma=\frac{1}{2 m^{4}}\left(\frac{49 m}{40 r}+\frac{29 m^{2}}{20 r^{2}}+\frac{19 m^{3}}{10 r^{3}}-\frac{203 m^{4}}{15 r^{4}}-\frac{436 m^{5}}{15 r^{5}}-\frac{184 m^{6}}{3 r^{6}}\right)
$$

where the integration constant has been chosen such that $\sigma$ is regular at the horizon $r=2 m$. The expression for $\tau$ can now be immediately obtained from (9a):

$$
\tau=-\frac{1}{2 m^{4}}\left(\frac{49 m}{40 r}+\frac{49 m^{2}}{20 r^{2}}+\frac{137 m^{3}}{30 r^{3}}+\frac{7 m^{4}}{15 r^{4}}-\frac{52 m^{5}}{15 r^{5}}-\frac{40 m^{6}}{3 r^{6}}\right)
$$

with the same boundary conditions as for $\sigma$.

The gravitational and inertial mass $m_{G}$ and $m_{I}$ are defined respectively as

$$
-g_{00} \approx 1-\frac{2 m_{G}}{r}+O\left(\frac{1}{r^{2}}\right) \quad g_{11} \approx 1+\frac{2 m_{I}}{r}+O\left(\frac{1}{r^{2}}\right)
$$


The two values coincide and are given by

$$
M \equiv m_{G}=m_{I}=m\left(1+\frac{49 \alpha^{2}}{80 m^{4}}\right)
$$

The mass of the black hole is therefore increased with respect to that of the Schwarzschild solution, or better, the horizon is shrunk to $r_{0}=2 M\left(1-49 \alpha^{2} / 80 M^{4}\right)$.

It is now easy to calculate the temperature $T$ of the hole, by requiring the regularity of the euclidean section of the metric at the horizon [21]. The result is

$$
\beta \equiv \frac{1}{T}=\left.8 \pi m\left[1+\alpha^{2}(\sigma-\tau)\right]\right|_{r=2 m}=8 \pi m\left[1+\frac{\alpha^{2}}{240 m^{4}}\right]
$$

Using (14) and (15) the temperature can be written in terms of the physical mass $M$ :

$$
T=\frac{1}{8 \pi M}\left(1+\frac{73 \alpha^{2}}{120 M^{4}}\right)
$$

which is higher than that of the Schwarzschild hole of equal mass.

Contrary to what happens in higher dimensions [17], the temperature is a monotonically decreasing function of the mass, and therefore no mechanism to prevent the complete evaporation of the black hole seems to be available in this case. As we shall see, the situation may change however for charged black holes.

The entropy is easily obtained from its definition in terms of the euclidean action (see section 4), and is given by:

$$
S=4 \pi M^{2}\left(1+\frac{\alpha}{M^{2}}+\frac{73 \alpha^{2}}{120 M^{4}}\right) .
$$

As is usual in higher derivative theories [22], it does not coincide with the area of the event horizon. It is, however, a positive definite function of $\mathrm{M}$.

\section{The purely magnetic case in the GHS gauge}

In order to study the effect of the Maxwell field it can be useful to write the metric in a different form. The reason is that an exact solution of the Einstein-Maxwell-dilaton system is known in the absence of the Gauss-Bonnet term [1,5], which has a very simple expression in a different "gauge" (i.e. coordinate system). For a magnetically charged hole it can be written as [1]:

$$
d s^{2}=-\lambda^{2} d t^{2}+\lambda^{-2} d r^{2}+R^{2} d \Omega^{2}
$$

where

$$
\lambda^{2}=1-\frac{2 m}{r} \quad R^{2}=r\left(r-\frac{\alpha q^{2}}{m}\right)
$$

and

$$
F=q \sin \theta d \theta \wedge d \varphi \quad \mathrm{e}^{-2 \Phi}=1-\frac{\alpha q^{2}}{m r}
$$


where $q$ is the magnetic charge ( $q$ is related to the physical charge $Q$ by $Q=\sqrt{\alpha / 4 \pi} q$ ). We start by discussing the expansion for the case in which the Maxwell term is present in the lagrangian, while the Gauss-Bonnet term is absent. In this way we shall compare the perturbative solution with the exact one and acquire some confidence with the GHS gauge.

We therefore write the metric in the form (19) and expand the metric functions around a Schwarzschild background as:

$$
\begin{gathered}
\lambda=\lambda_{0}\left(1+\alpha \psi_{1}+\alpha^{2} \psi_{2}+\ldots\right) ; \quad R=r+\alpha \rho_{1}+\alpha^{2} \rho_{2}+\ldots \\
\Phi=\alpha \phi_{1}+\alpha^{2} \phi_{2}+\ldots,
\end{gathered}
$$

where $\lambda_{0}=\left(1-\frac{2 m}{r}\right)^{1 / 2}$ and $\psi_{i}, \rho_{i}$ and $\phi_{i}$ are functions of $r$.

The equation $(2 \mathrm{c})$ is solved by the ansatz

$$
F_{i j}=\frac{q}{R^{2}} \epsilon_{i j}
$$

The equation for the dilaton becomes at first order in $\alpha$ :

$$
\left[r(r-2 m) \phi_{1}^{\prime}\right]^{\prime}=-\frac{q^{2}}{r^{2}}
$$

which, up to an integration constant yields $\phi_{1}=\frac{q^{2}}{2 m r}$. The other equations are given at order $\alpha$ by

$$
\begin{aligned}
\rho_{1}^{\prime \prime} & =0 \\
{\left[(r-2 m) \psi_{1}\right]^{\prime} } & =-\frac{m}{r^{2}} \rho_{1}-\frac{q^{2}}{2 r^{2}}
\end{aligned}
$$

We impose the boundary conditions that $\rho_{1} \rightarrow$ const, $\psi_{1} \rightarrow 0$ at infinity. We are still free to choose the boundary conditions at $r=2 \mathrm{~m}$. Changing the boundary conditions at $r=2 \mathrm{~m}$ yields a reparametrization of the solutions, but no change in their physical properties: in particular, the relations between the physical quantities, like mass, temperature and entropy, are independent of the parametrization. The simplest choice for the following calculations is to choose for $\rho_{1}$, which must be constant in view of $(22 \mathrm{~b})$ and the boundary conditions at infinity, the value $\rho_{1}=-\frac{q^{2}}{2 m}$, so that the r.h.s. of $(22 \mathrm{c})$ vanishes. This choice permits us to have $\psi_{1}=0$.

We can now evaluate the second order corrections. For vanishing $\psi_{1}$ the equations are given by:

$$
\begin{gathered}
{\left[r(r-2 m) \phi_{2}^{\prime}\right]^{\prime}=2 \frac{r-2 m}{r} \rho_{1} \phi_{1}^{\prime}+\frac{2 q^{2}}{r^{2}}\left(\phi_{1}+\frac{2 \rho_{1}}{r}\right)=\frac{q^{4}}{2 m^{2}}\left(\frac{1}{r^{2}}-\frac{4 m}{r^{3}}\right)} \\
\rho_{2}^{\prime \prime}=-r \phi_{1}^{\prime 2}=-\frac{q^{4}}{4 m^{2} r^{3}}
\end{gathered}
$$




$$
\left[(r-2 m) \psi_{2}\right]^{\prime}=-\frac{r-2 m}{2} \rho_{2}^{\prime \prime}-\frac{r-m}{r} \rho_{2}^{\prime}-\frac{m}{r^{2}} \rho_{2}+\frac{2 m}{r^{3}} \rho_{1}^{2}+\frac{q^{2}}{r^{2}}\left(\phi_{1}+\frac{2 \rho_{1}}{r}\right)=0
$$

The solutions satisfying the boundary conditions stated above are easily seen to be:

$$
\phi_{2}=\frac{q^{4}}{4 m^{2} r^{2}}, \quad \rho_{2}=-\frac{q^{4}}{8 m^{2} r}, \quad \psi_{2}=0
$$

It is now straightforward to compare the expansion with the exact result (19): it turns out that it coincides with the expansion of the exact solution in powers of $\alpha$ :

$$
\begin{gathered}
\Phi=-\frac{1}{2} \ln \left(1-\frac{\alpha q^{2}}{m r}\right) \sim \frac{\alpha q^{2}}{2 m r}+\frac{\alpha^{2} q^{4}}{4 m^{2} r^{2}} \\
\lambda=\lambda_{0}=\sqrt{1-\frac{2 m}{r}} \\
R=\sqrt{r\left(r-\frac{\alpha q^{2}}{m}\right)} \sim r-\frac{\alpha q^{2}}{2 m}-\frac{\alpha^{2} q^{4}}{8 m^{2} r}
\end{gathered}
$$

The physical properties of this solution are discussed in $[1,8]$ and we shall not report them here. It may be worth noticing, however, that the values of the temperature and entropy of the hole in this approximation, obtained up to second order in $\alpha$ with the methods described in the next section, turn out to coincide with the exact result [8]:

$$
T=\frac{1}{8 \pi M}, \quad S=4 \pi\left(M^{2}-\frac{\alpha q^{2}}{2}\right)
$$

where $M=m$ is the physical mass, as is evident from (25b).

\section{Solutions of the full order $\alpha^{\prime}$ effective action}

We now consider the more interesting case where both the Maxwell and Gauss-Bonnet terms are present. As we shall see, whereas the Maxwell term contributes already at first order to the metric functions, the Gauss-Bonnet terms contributes only at second order. This fact gives some justification to the interpretation of the GHS solution as a first order approximation for the metric. However, this is not true for the scalar field, to which the Gauss-Bonnet terms contribute already at first order. With the ansatz (21) for $F$, in the notation of the previous section, the field equations (2) become at order $\alpha$ :

$$
\begin{aligned}
{\left[r(r-2 m) \phi_{1}^{\prime}\right]^{\prime} } & =-\frac{q^{2}}{r^{2}}+\frac{24 m^{2}}{r^{4}} \\
\rho_{1}^{\prime \prime} & =0 \\
{\left[(r-2 m) \psi_{1}\right]^{\prime} } & =-\frac{m}{r^{2}} \rho_{1}-\frac{q^{2}}{2 r^{2}}
\end{aligned}
$$


Again, we impose boundary conditions such that the metric is asymptotically flat and exploit the remaining freedom to fix $\psi_{1}=0$. Integration of (27) yields:

$$
\begin{gathered}
\phi_{1}=-\frac{1}{m}\left(\frac{2-q^{2}}{2 r}+\frac{m}{r^{2}}+\frac{4 m^{2}}{3 r^{3}}\right) \\
\rho_{1}=-\frac{q^{2}}{2 m}, \quad \psi_{1}=0
\end{gathered}
$$

The dilatonic charge is now given at first order by $\frac{2-q^{2}}{2 m} \alpha$. The long range potential driven by the scalar field can therefore be attractive or repulsive depending on the value of $q^{2}$.

We go now to second order in $\alpha$. The field equations become:

$$
\begin{gathered}
{\left[r(r-2 m) \phi_{2}^{\prime}\right]^{\prime}=2 \frac{r-2 m}{r} \rho_{1} \phi_{1}^{\prime}+\frac{2 q^{2}}{r^{2}}\left(\phi_{1}+2 \frac{\rho_{1}}{r}\right)-\frac{48 m^{2}}{r^{4}}\left(\phi_{1}+\frac{\rho_{1}}{r}\right)} \\
=\frac{2 q^{2}-q^{4}}{2 m^{2} r^{2}}+2 \frac{q^{2}+q^{4}}{m r^{3}}+\frac{2 q^{2}}{r^{4}}+16 m \frac{9+q^{2}}{3 r^{5}}+\frac{48 m^{2}}{r^{6}}+\frac{64 m^{3}}{r^{7}} \\
\rho_{2}^{\prime \prime}=-r \phi_{1}^{\prime 2}+\frac{8 m}{r^{2}} \phi_{1}^{\prime \prime} \\
=-\left[\frac{\left(q^{2}-2\right)^{2}}{4 m^{2} r^{3}}+2 \frac{2-q^{2}}{m r^{4}}+4 \frac{7-3 q^{2}}{r^{5}}+\frac{64 m}{r^{6}}+\frac{144 m^{2}}{r^{7}}\right] \\
{\left[(r-2 m) \psi_{2}\right]^{\prime}=-\frac{r-2 m}{2} \rho_{2}^{\prime \prime}-\frac{r-m}{r} \rho_{2}^{\prime}-\frac{m}{r^{2}} \rho_{2}+\frac{2 m}{r^{3}} \rho_{1}^{2}} \\
+\frac{q^{2}}{r^{2}}\left(\phi_{1}+\frac{2 \rho_{1}}{r}\right)+4 m\left(\frac{r-2 m}{r^{2}} \phi_{1}^{\prime \prime}-2 \frac{r-3 m}{r^{3}} \phi_{1}^{\prime}\right) \\
=2 \frac{1-2 q^{2}}{3 m r^{3}}-\frac{11-5 q^{2}}{r^{4}}+8 m \frac{1-25 q^{2}}{15 r^{5}}+\frac{544 m^{3}}{5 r^{7}}
\end{gathered}
$$

It is no longer possible to have a vanishing $\psi_{2}$. We therefore choose the boundary conditions such that $\lambda^{2} \approx 1-\frac{2 m}{r}+O\left(\frac{1}{r^{2}}\right)$ at infinity, so that $m$ is by definition the physical mass $M$ of the hole. The solutions of (29) are:

$$
\begin{gathered}
\phi_{2}=-\left[\frac{73-45 q^{2}}{60 m^{3} r}+\frac{73-15 q^{2}-15 q^{4}}{60 m^{2} r^{2}}+\frac{73}{45 m r^{3}}+\frac{73+5 q^{2}}{30 r^{4}}+\frac{112 m}{75 r^{5}}+\frac{8 m^{2}}{9 r^{6}}\right] \\
\rho_{2}=-\left[\frac{\left(q^{2}-2\right)^{2}}{8 m^{2} r}+\frac{2-q^{2}}{3 m r^{2}}+\frac{7-3 q^{2}}{3 r^{3}}+\frac{16 m}{5 r^{4}}+\frac{24 m^{2}}{5 r^{5}}\right] \\
\tilde{\psi}_{2} \equiv(r-2 m) \psi_{2}=-\left[\frac{1-2 q^{2}}{3 m r^{2}}-\frac{11-5 q^{2}}{3 r^{3}}+\frac{\left(2-50 q^{2}\right) m}{15 r^{4}}+\frac{272 m^{3}}{15 r^{6}}\right]
\end{gathered}
$$

The metric functions are therefore:

$$
\begin{aligned}
R^{2} & =r^{2}+2 \alpha r \rho_{1}+\alpha^{2}\left(2 r \rho_{2}+\rho_{1}^{2}\right) \\
& =r^{2}-\frac{\alpha q^{2}}{m} r-\alpha^{2}\left[\frac{1-q^{2}}{m^{2}}+2 \frac{2-q^{2}}{3 m r}+2 \frac{7-3 q^{2}}{3 r^{2}}+\frac{32 m}{5 r^{3}}+\frac{48 m^{2}}{5 r^{4}}\right]
\end{aligned}
$$




$$
\begin{aligned}
\lambda^{2} & =1-\frac{2 m}{r}+2 \alpha^{2} \frac{\tilde{\psi}_{2}}{r} \\
& =1-\frac{2 m}{r}-2 \alpha^{2}\left[\frac{1-2 q^{2}}{3 m r^{3}}-\frac{11-5 q^{2}}{3 r^{4}}+\frac{\left(2-50 q^{2}\right) m}{15 r^{5}}+\frac{272 m^{3}}{15 r^{7}}\right]
\end{aligned}
$$

They are sketched in Figure 1, for different values of $q$. For $q=0$ one obtains of course the solution discussed in section 2 , but in different coordinates. In particular, in the present coordinates the horizon is at $r_{+}=2 m\left(1-\alpha^{2} \tilde{\psi}_{2}(2 m)\right)=2 m\left(1-\frac{1+2 q^{2}}{12 m^{4}} \alpha^{2}\right)$. We notice that the coordinate $r$ should not be identified with the physical radial coordinate, which is rather given by $R$. Actually, $r$ must vary in the range $r \geq r_{-}$, where $r_{-} \approx \frac{\alpha q^{2}}{m}$ is the greatest zero of $R^{2}$, which corresponds to a singular surface. When $r_{-}$becomes greater than $r_{+}$(for $\left.\alpha q^{2}>2 m^{2}(1+7 \alpha / 12)\right)$, one has a naked singularity. This situation is, however, beyond the range of validity of our approximation.

It is now easy to calculate the temperature of the hole. The inverse temperature is defined as the periodicity of the time coordinate which renders the euclidean section of the solution regular and is given by [21]:

$$
\beta=\left.4 \pi \sqrt{g_{00} g_{11}}\left[\frac{d}{d t} g_{00}\right]^{-1}\right|_{r=2 m}
$$

In our coordinates eqn.(32) becomes at order $\alpha^{2}$ :

$$
\beta=8 \pi m\left[1-\alpha^{2}\left(\frac{\tilde{\psi}_{2}(2 m)}{m}-2 \tilde{\psi}_{2}^{\prime}(2 m)\right)\right]=8 \pi m\left(1-\alpha^{2} \frac{73-45 q^{2}}{120 m^{4}}\right)
$$

It is interesting to notice that the temperature of the black hole, which is given by

$$
T=\frac{1}{8 \pi M}\left(1+\alpha^{2} \frac{73-45 q^{2}}{120 M^{4}}\right)
$$

has no order $\alpha$ corrections (we recall that the physical mass $M=m$ in these coordinates). The behaviour of $T$ as a function of $M$ is displayed in Figure 2a for several values of $q$.

It is remarkable the fact that the temperature is no longer independent of the charge, as in the GHS solution, but has a very different behaviour depending on the values of $q$. In particular, if $q^{2}>73 / 45$, the temperature vanishes for $M^{4}=\alpha^{2}\left(\frac{3}{8} q^{2}-\frac{73}{120}\right)$. The hole may therefore reach during its evaporation a stable groundstate with nonvanishing values of mass and charget. Unfortunately, however, for such values of $q$ and $M$ our approximation breaks down and therefore it is not possible to discuss the causal structure of the solution. In particular, we are not able to establish if the states with vanishing temperature correspond to regular horizons or naked singularities.

$\dagger$ The implications for cosmology of a model with similar behaviour of $T$ have been recently discussed in [23] and, in their notation, $m_{r e l}^{4} \propto\left(\frac{3 q^{2}}{8}-\frac{73}{120}\right) m_{P l}^{4}$. 
The entropy of the hole can be calculated by evaluating the euclidean action [24]:

$$
I_{E}=-\frac{1}{16 \pi} \int_{M}\left[\mathcal{R}-2(\nabla \Phi)^{2}+\alpha \mathrm{e}^{-2 \Phi}\left(\mathcal{S}-F^{2}\right)\right] d V-\frac{1}{8 \pi} \int_{\partial M}\left(K-K_{0}+\mathcal{Q}\right) d \Sigma
$$

where the boundary integral includes a term $\mathcal{Q}$ coming from the Gauss-Bonnet part of the action. This term, which is proportional to the Chern-Simons form on the boundary [22], gives vanishing contribution in four dimensions.

By using the equations of motion (2) and neglecting $O\left(\alpha^{3}\right)$ terms, $I_{E}$ can therefore be reduced to the form:

$$
\begin{aligned}
I_{E} & =-\frac{\alpha}{16 \pi} \int_{M} \mathrm{e}^{-2 \Phi}\left(\mathcal{S}-F^{2}\right)-\frac{1}{8 \pi} \int_{\partial M}\left(K-K_{0}\right)=-\frac{1}{8 \pi} \int_{M} \nabla^{2} \Phi-\frac{1}{8 \pi} \int_{\partial M}\left(K-K_{0}\right) \\
& =\frac{1}{2} \beta m\left(1-\alpha \frac{2-q^{2}}{2 m^{2}}-\alpha^{2} \frac{73-45 q^{2}}{60 m^{4}}\right)=4 \pi m^{2}\left(1-\alpha \frac{2-q^{2}}{2 m^{2}}-\alpha^{2} \frac{73-45 q^{2}}{40 m^{4}}\right)
\end{aligned}
$$

The mass $M$ of the hole can be defined as $M=\left.\frac{\partial I}{\partial \beta}\right|_{q}$, where

$$
\left.\frac{\partial}{\partial \beta}\right|_{q}=\frac{\partial m}{\partial \beta} \frac{\partial}{\partial m}=\frac{1}{8 \pi}\left(1-\alpha^{2} \frac{73-45 q^{2}}{40 m^{4}}\right) \frac{\partial}{\partial m}
$$

One has therefore, by (36),

$$
M=\frac{1}{2}\left(1-\alpha^{2} \frac{73-45 q^{2}}{40 m^{4}}\right) \frac{\partial}{\partial m}\left(1-\alpha^{2} \frac{73-45 q^{2}}{40 m^{4}}\right)=m,
$$

as expected. The entropy is then given by:

$$
S=\beta \frac{\partial I}{\partial \beta}-I=4 \pi M^{2}\left(1+\alpha \frac{2-q^{2}}{2 M^{2}}+\alpha^{2} \frac{73-45 q^{2}}{120 M^{4}}\right)
$$

Its behaviour as a function of $M$ is displayed in figure $2 \mathrm{~b}$.

We note that the entropy has a zero for $\alpha q^{2}=2 M^{2}\left(1+\frac{\alpha}{4 M^{2}}+\frac{101 \alpha^{2}}{240 M^{4}}\right)$. For the exact GHS solution the zero of the entropy corresponds to the case of extremal black holes [8]. By comparing (34) and (39) it is easy to see that, for positive $\alpha$, if $q^{2}>2$ the zero of the entropy occurs for values of $M$ greater than those for which $T=0$, while for $q^{2}<2$ one has the opposite situation. It seems therefore that when the Gauss-Bonnet terms are taken into account, for small or zero charge the situation is more similar in this respect to the usual black hole thermodynamics, while for greater charge one has physical properties more similar to those of the GHS solution [8]. It may be useful to remark that this critical value of $q^{2}$ is precisely that for which the long range scalar potential change from attractive to repulsive at first order in $\alpha$ (see eq.(28a)).

Of course, we are considering only the lower order corrections to $T$ and $S$. When higher terms of the expansion are taken into account the situation may change. In particular, for small values of $M$ our approximation is not reliable. Our results should be essentially unchanged, however, for large values of mass and charge. 


\section{Final remarks}

We have studied the effect of the inclusion of the Gauss-Bonnet corrections induced by string theory on the spherically symmetric solutions representing the charged dilatonic black holes obtained in $[1,5]$. The physical properties of the solution resemble those of the GHS solution for large charge. For small or vanishing charge the situation looks similar to the more conventional theories.

In particular, the Gauss-Bonnet term induces $O\left(\alpha^{2}\right)$ charge-dependent corrections to the temperature. Moreover, for $q^{2}>\frac{73}{45}$, our stringy black hole has vanishing temperature for a finite positive value of $M$. Our analysis thus illustrates the importance of the higher order curvature correction for the thermodynamics of an evaporating classical black hole.

We have considered the string effective action up to order $\alpha^{\prime}$. The results obtained are not essentially affected if one takes into account the order $\alpha^{\prime 2}$ corrections to the effective action, as given in [26]. The new terms, in fact, give contributions of order $\alpha^{\prime 2}$ to $\Phi$ and of order $\alpha^{\prime 3}$ to the metric fields. The formula (34) for the temperature, in particular, is corrected only by third order terms.

Our results may be extended to the case of electric charge. Unfortunately, due to the presence of the Gauss-Bonnet term, the electromagnetic duality which allows one to obtain immediately the electrically charged solution from the magnetic one [3] is no longer a symmetry of the field equations. The calculation should therefore be started ab initio. Analogous considerations hold for the dyonic case, with the further complication that the axion field should also be taken into account in this case [13]. These problems are currently under investigation.

Other interesting perspectives are given by the study of massive dilatons. The dilaton potential generated by the compactification mechanism may induce some modifications in the properties of the black holes. Some results have been obtained in ref. [27], in the absence of the Gauss-Bonnet term.

\section{Acknowledgements}

N.R.S. wishes to thank the Royal Society for a European fellowship. The work of S.M. was supported by a MRST fellowship.

\section{Appendix}

In this appendix we rederive the results of section 5 in a more conventional gauge. We adopt the metric form (7) and expand the metric functions as:

$$
\nu=\nu_{0}+\alpha \tau_{1}+\alpha^{2} \tau_{2} \ldots \quad \mu=\mu_{0}+\alpha \sigma_{1}+\alpha^{2} \sigma_{2}
$$

where $\nu_{0}$ and $\mu_{0}$ are the values of $\mu$ and $\nu$ in the Schwarzschild background of mass $m$. Due to the presence of the Maxwell field, order $\alpha$ corrections to the metric functions must also be taken into account. The parameter $m$ and the radial coordinate $r$ used in this appendix do not coincide of course with those of section 5 . The relations between the physical quantities (mass, temperature, entropy...) are nevertheless the same in the two gauges.

With the ansatz (21) for $F$, the field equation (27a) for $\phi_{1}$ is unchanged in this gauge, and the solution is still given by $(28 \mathrm{a})$. 
The gravitational equations become at first order:

$$
\begin{gathered}
\tau_{1}^{\prime}+\sigma_{1}^{\prime}=0 \\
(r-2 m)\left(\sigma_{1}^{\prime}-\tau_{1}^{\prime}\right)+2 \sigma_{1}=\frac{q^{2}}{r^{2}}
\end{gathered}
$$

By requiring asymptotic flatness and regularity at the horizon, the solutions of (A.2) are given uniquely by:

$$
\sigma_{1}=-\tau_{1}=\frac{q^{2}}{4 m r}
$$

Using (A.3) the field equation for the second order corrections to the scalar field can be written as:

$$
\left[r(r-2 m) \phi_{2}^{\prime}\right]^{\prime}=\frac{(r-2 m) q^{2}}{2 m r^{2}} \phi_{1}^{\prime \prime}+\frac{q^{2}}{2 m r^{2}} \phi_{1}^{\prime}+\frac{2}{r^{4}}\left[q^{2}-\frac{48 m^{2}}{r^{2}}\right] \phi_{1}+\frac{12 q^{2}}{r^{6}}\left[1-\frac{4 m}{r}\right]
$$

which has solution:

$$
\begin{aligned}
\phi_{2}= & -\frac{1}{360 m^{4}}\left[\left(438-360 q^{2}+45 q^{4}\right) \frac{m}{r}+\left(438-270 q^{2}\right) \frac{m^{2}}{r^{2}}+\left(548-240 q^{2}\right) \frac{m^{3}}{r^{3}}\right. \\
& \left.+\left(876-660 q^{2}\right) \frac{m^{4}}{r^{4}}+\frac{5376}{10} \frac{m^{5}}{r^{5}}+320 \frac{m^{6}}{r^{6}}\right]
\end{aligned}
$$

where as usual we require regularity at the horizon and asymptotic flatness. The gravitational equations are at second order:

$$
\begin{gathered}
\tau_{2}^{\prime}+\sigma_{2}^{\prime}=r \phi_{1}^{\prime 2}-\frac{8 m}{r^{2}} \phi_{1}^{\prime \prime} \\
\sigma_{2}^{\prime}-\tau_{2}^{\prime}+\frac{2 \sigma_{2}}{r-2 m}=-\frac{8 m}{r^{2}} \phi_{1}^{\prime \prime}+\frac{16 m(r-3 m)}{r^{3}(r-2 m)} \phi_{1}^{\prime}-\frac{2 q^{2}}{r^{2}(r-2 m)} \phi_{1}-\frac{(r-4 m) q^{4}}{8 m^{2} r^{3}(r-2 m)}
\end{gathered}
$$

With the usual boundary conditions their solutions are:

$$
\begin{aligned}
\tau_{2}= & -\frac{1}{480 m^{4}}\left[\left(294-30 q^{2}-15 q^{4}\right) \frac{m}{r}+\left(588-60 q^{2}\right) \frac{m^{2}}{r^{2}}+\left(1096-200 q^{2}\right) \frac{m^{3}}{r^{3}}\right. \\
& \left.+\left(112+560 q^{2}\right) \frac{m^{4}}{r^{4}}-832 \frac{m^{5}}{r^{5}}-3200 \frac{m^{6}}{r^{6}}\right] \\
\sigma_{2}= & \frac{1}{480 m^{4}}\left[\left(294-30 q^{2}-15 q^{4}\right) \frac{m}{r}+\left(348+180 q^{2}-60 q^{4}\right) \frac{m^{2}}{r^{2}}+\left(456+120 q^{2}\right) \frac{m^{3}}{r^{3}}\right. \\
& \left.-\left(3248-2000 q^{2}\right) \frac{m^{4}}{r^{4}}-6976 \frac{m^{5}}{r^{5}}-14720 \frac{m^{6}}{r^{6}}\right]
\end{aligned}
$$

The physical mass $M$ of the hole, defined as in (13) is given by:

$$
M \equiv m_{G}=m_{I}=m\left[1+\alpha \frac{q^{2}}{4 m^{2}}+\alpha^{2} \frac{294-30 q^{2}-15 q^{4}}{480 m^{4}}\right]
$$


Inverting, one can write the parameter $m$ as a function of the physical mass:

$$
m=M\left[1-\alpha \frac{q^{2}}{4 M^{2}}-\alpha^{2} \frac{294-30 q^{2}+15 q^{4}}{480 M^{4}}\right]
$$

One can now deduce the temperature from (32): one has, up to second order:

$$
\begin{aligned}
\beta^{-1} \equiv T & =\left.\frac{1}{8 \pi m}\left[1+\alpha\left(\tau_{1}-\sigma_{1}\right)+\alpha^{2}\left(\tau_{2}-\sigma_{2}\right)+\frac{\alpha^{2}}{2}\left(\tau_{1}-\sigma_{1}\right)^{2}\right]\right|_{r=2 m} \\
& =\frac{1}{8 \pi m}\left[1-\alpha \frac{q^{2}}{4 m^{2}}-\alpha^{2} \frac{2+150 q^{2}-45 q^{4}}{480 m^{4}}\right]
\end{aligned}
$$

Using (A.9) one recovers the result of section 5 for the temperature in terms of the physical mass:

$$
T=\frac{1}{8 \pi M}\left[1+\alpha^{2} \frac{73-45 q^{2}}{120 M^{4}}\right]
$$

Analogously, following the procedure of section 5, one can evaluate the entropy; the euclidean action turns out to be:

$$
\begin{aligned}
I_{E} & =\frac{\beta}{2}\left[m-\alpha \frac{4-3 q^{2}}{4 m}-\alpha^{2} \frac{290-450 q^{2}+75 q^{4}}{480 m^{3}}\right] \\
& =4 \pi M^{2}\left[1-\alpha \frac{2-q^{2}}{2 M^{2}}-\alpha^{2} \frac{73-45 q^{2}}{40 M^{4}}\right]
\end{aligned}
$$

which is the result of section 5. The value of the entropy is therefore given by (39).

\section{References}

[1] D. Garfinkle, G.T. Horowitz, A. Strominger, Phys. Rev. D43, 3140 (1991)

[2] B.A. Campbell, M.J. Duncan, N. Kaloper, K.A. Olive, Phys. Lett. 251B, 34 (1990)

[3] A. Shapere, S. Trivedi, F. Wilczek, Mod. Phys. Lett. A6, 2677 (1991)

[4] J.D. Bekenstein, Phys. Rev. D5,1239 (1972)

J.E. Chase, Comm. Math. Phys. 19, 276 (1970)

[5] G.W. Gibbons, K. Maeda, Nucl. Phys. B298, 741 (1988)

[6] A. Sen, preprint TIFR-TH-92-90, TIFR-TH-92-41 (1992)

[7] J. Preskill, P. Schwarz, A. Shapere, S. Trivedi, F. Wilczek, Mod. Phys. Lett. A6, 2353 (1991)

[8] C.F.E. Holzhey, F. Wilczek, Nucl. Phys. B380, 447 (1992)

[9] S.B. Giddings, A. Strominger, Phys. Rev. D46, 627 (1992)

[10] M.J. Bowick, S.B. Giddings, J.A. Harvey, G.T. Horowitz, A. Strominger, Phys. Rev. Lett. 61, 2823 (1988)

[11] S. Mignemi, N.R. Stewart, preprint, in press, Phys. Lett. B (1992)

[12] B.A. Campbell, M.J. Duncan, N. Kaloper, K.A. Olive, Phys. Lett. 263B, 364 (1990)

B.A. Campbell, N. Kaloper, K.A. Olive, Phys. Lett. 285B, 199 (1990)

K. Lee, E.J. Weinberg, Phys. Rev. D44, 3159 (1991) 
[13] B.A. Campbell, M.J. Duncan, N. Kaloper, K.A. Olive, Nucl. Phys. B351,778 (1991)

[14] D. Lovelock, J. Math. Phys. 12, 498 (1991)

[15] B. Zwiebach, Phys. Lett. 165B, 315 (1985)

[16] D.G. Boulware, S. Deser, Phys. Lett. 175B, 409 (1986)

[17] C.G. Callan, R.C. Myers, M.J. Perry, Nucl. Phys. B311, 673 (1988)

[18] A. Tomimatsu, Prog. Theor. Phys. 79, 86 (1988)

[19] E. Poisson, Class. Quantum Grav. 8, 639 (1991)

[20] L. Sokolowski, Class. Quantum Grav. 6, 2045 (1989)

[21] J.W. York, Phys. Rev. D31, 775 (1985)

[22] B. Whitt, Phys. Rev. D38, 3000 (1988) R.C. Myers, J.Z. Simon, Phys. Rev. D38, 2434 (1988)

[23] J.D. Barrow, E.J. Copeland, A.R. Liddle, preprint Sussex-AST-92/3-1 (1992)

[24] S.W. Hawking, in "General Relativity: an Einstein centenary survey", eds. S.W. Hawking and W. Israel (Cambridge Un. Press 1979)

[25] R.C. Myers, Phys. Rev. D36, 392 (1987)

F. Müller-Hoissen, Nucl. Phys. B337, 709 (1990)

[26] D.J. Gross, J.H. Sloane, Nucl. Phys. B291, 41 (1987)

[27] R. Gregory, J.A. Harvey, preprint EFI-92-49 (1992) 


\section{Figure captions}

Fig. 1a) The metric function $R^{2}$ for $\alpha=1, M=10$.

Fig. 1b) The metric function $\lambda^{2}$ for $\alpha=1, M=10$.

Fig. 1c) The scalar field $\Phi$ for $\alpha=1, M=10$.

Fig. 2a) The temperature as a function of the mass for several values of $q(\alpha=1)$. The behaviour changes drastically for $q^{2}>\frac{73}{45}$.

Fig. 2b) The temperature as a function of the mass for several values of $q(\alpha=1)$. Also in this case the behaviour is very different depending on $q^{2}$ being greater, equal or less than $\frac{73}{45}$. 